\title{
ON NONAUTONOMOUS DISCRETE DYNAMICAL SYSTEMS DRIVEN BY MEANS
}

\author{
RAGHIB M. ABU-SARIS
}

Received 27 March 2006; Revised 13 June 2006; Accepted 14 June 2006

We investigate the asymptotic behavior of nonautonomous discrete dynamical systems governed by the system of difference equations (recursive equations): $y_{j}(n+1)=F_{j}(n$, $\mathbf{y}(n)) ; j=1, \ldots, k, n=0,1,2, \ldots$, where $\mathbf{y}(n)=\left(y_{1}(n), \ldots, y_{k}(n)\right) \in \mathbb{R}^{k}, \mathbf{y}(0)=\mathbf{x}$, and $F_{j}(n, \cdot)$ is a mean of $k(\geq 2)$ positive real numbers, that is, a real-valued function satisfying the internality property $\min (\mathbf{x}) \leq F_{j}(n, \mathbf{x}) \leq \max (\mathbf{x})$.

Copyright (c) 2006 Raghib M. Abu-Saris. This is an open access article distributed under the Creative Commons Attribution License, which permits unrestricted use, distribution, and reproduction in any medium, provided the original work is properly cited.

\section{Introduction}

In this research, we are concerned with the long-term behavior of discrete dynamical systems driven by means, namely,

$$
y_{j}(n+1)=F_{j}(n, \mathbf{y}(n)) ; \quad j=1, \ldots, k, n=0,1,2, \ldots,
$$

where $\mathbf{y}(n)=\left(y_{1}(n), \ldots, y_{k}(n)\right), \mathbf{y}(0)=\mathbf{x}$, and for each $j, F_{j}(n, \cdot)$ is a mean of $k(\geq 2)$ positive real numbers, that is, a real-valued function satisfying the internality property

$$
\min (\mathbf{x}) \leq F_{j}(n, \mathbf{x}) \leq \max (\mathbf{x})
$$

$A$ mean $M$ is strict if

$$
\min (\mathbf{x})<\max (\mathbf{x}) \Longrightarrow \min (\mathbf{x})<M(\mathbf{x})<\max (\mathbf{x})
$$

and it is said to be isotone if the functions $M\left(\cdot, x_{2}, \ldots, x_{k}\right), \ldots, M\left(x_{1}, \ldots, x_{k-1}, \cdot\right)$ are nondecreasing.

There is a sizable number of means in the literature. For example

(i) the (weighted) arithmetic means $A(\mathbf{x})=\sum_{j=1}^{k} w_{j} x_{j}, w_{j} \geq 0, \sum_{j=1}^{k} w_{j}=1$;

(ii) the (weighted) geometric means $G(\mathbf{x})=\prod_{j=1}^{k} x_{j}^{v_{j}}, v_{j} \geq 0, \sum_{j=1}^{k} v_{j}=1$; 
(iii) the Holder means $H_{p}(\mathbf{x})=\left[\sum_{j=1}^{k} w_{j} x_{j}^{p}\right]^{1 / p}, p>0, w_{j} \geq 0, \sum_{j=1}^{k} w_{j}=1$;

(iv) the Lehmer means $L_{p}(\mathbf{x})=\sum_{j=1}^{k} x_{j}^{p} / \sum_{j=1}^{k} x_{j}^{p-1}, p>1$, and so forth. For more information on means and related results, we refer the reader to [2-4] and the references cited therein.

The dynamical system described in (1.1) is, also, known in literature as compounding [3] or iteration [9] of means. Markov chains, which are basically compounding of arithmetic means, illustrate, in a clear way, the importance of such problem in modeling and applications. Also, Gauss arithmetic-geometric mean, one of the jewels of the classical analysis, connects iteration of means to special functions and integrability of difference equations.

Most of the literature related to compounding of means deal with time-invariant (autonomous) dynamical systems. It is our objective in this research to extend these results to time-variant (nonautonomous) dynamical systems driven by means. The presence of time accounts for environmental fluctuations, seasonal change, and so forth [5]. Our main results are established in Section 2. We present some applications of these results in Section 3, and conclude in Section 4 by formulating a conjecture about the limit sets of the dynamical systems under study.

\section{Main results}

In this section, we state and prove our main results. The following lemma is important in its own right, and will play a key role in the sequel.

Lemma 2.1. Let $\mathscr{Y}=\{\mathbf{y}(n)\}_{n=0}^{\infty}$ be a solution of (1.1) with $\mathbf{y}(0)=\mathbf{x}$. Then, there exist real numbers $L$ and $U$ with $L \leq U$ such that limit set $w(\mathbf{x})$ is a subset of the intersection of the levels sets $\min (\tilde{\mathbf{x}})=L$ and $\max (\tilde{\mathbf{x}})=U$. In particular, if $k=2$, then 9 can have at most two limit points.

Proof. First, let $L_{n}=\min (\mathbf{y}(n))$ and $U_{n}=\max (\mathbf{y}(n))$. Then for all $n \geq 0$,

$$
\begin{aligned}
L_{n+1} & =\min (\mathbf{y}(n+1))=\min \left(F_{1}(n, \mathbf{y}(n)), \ldots, F_{k}(n, \mathbf{y}(n))\right) \\
& \geq \min \left(L_{n}, \ldots, L_{n}\right)=L_{n}, \\
U_{n+1} & =\max (\mathbf{y}(n+1))=\max \left(F_{1}(n, \mathbf{y}(n)), \ldots, F_{k}(n, \mathbf{y}(n))\right) \\
& \leq \max \left(U_{n}, \ldots, U_{n}\right)=U_{n} .
\end{aligned}
$$

Therefore, by monotonic convergence theorem, the sequences $\left\{L_{n}\right\}_{n=0}^{\infty}$ and $\left\{U_{n}\right\}_{n=0}^{\infty}$ converge say to $L$ and $U$, respectively.

Now, if $\mathbf{c}$ is a limit point and $\left\{\mathbf{y}\left(n_{i}\right)\right\}_{i=0}^{\infty}$ is a subsequence that converges to $\mathbf{c}$, then by the continuity of the min and the max functions,

$$
L=\lim _{i \rightarrow \infty} \min \left(\mathbf{y}\left(n_{i}\right)\right)=\min (\mathbf{c}), \quad U=\lim _{i \rightarrow \infty} \max \left(\mathbf{y}\left(n_{i}\right)\right)=\max (\mathbf{c})
$$

The last part of the assertion follows from the fact that the level curves $\min \left(x_{1}, x_{2}\right)=L$ and $\max \left(x_{1}, x_{2}\right)=U$ can intersect in at most two points. This completes the proof. 
Theorem 2.2. Suppose that for each $j, F_{j}(n, \cdot)$ is continuous and converges to the continuous mean $M_{j}(\cdot)$. If at least $k-1$ of the means $M_{j}$ 's are strict, then every solution $\{\mathbf{y}(n)\}_{n=0}^{\infty}$ converges to $\mu(\mathbf{x}) \mathbf{e}$, where $\mathbf{e}=(1, \ldots, 1)$. Furthermore, $\mu$ is a continuous mean.

Proof. First, recall the definition of $L$ and $U$ from the proof of Lemma 2.1, and observe that by Weierstrass boundedness theorem, the convergence of $F_{j}(n, \cdot)$ to $M_{j}(\cdot)$ is uniform on any compact subset of $\mathbb{R}^{k}$, in particular on $\left[L_{0}, U_{0}\right]^{k}$. Therefore (see Theorem 2 and its proof in [8]), if $\left\{\mathbf{y}\left(n_{i}\right)\right\}_{i=0}^{\infty}$ is a subsequence that converges to a limit point $\mathbf{c}$, then $\left\{F_{j}\left(n_{i}, \mathbf{y}\left(n_{i}\right)\right)\right\}_{i=0}^{\infty}$ converges $M_{j}(\mathbf{c})$.

Now, since

$$
\begin{aligned}
& \min (\mathbf{c})=L=\min \left(M_{1}(\mathbf{c}), \ldots, M_{k}(\mathbf{c})\right), \\
& \max (\mathbf{c})=U=\max \left(M_{1}(\mathbf{c}), \ldots, M_{k}(\mathbf{c})\right),
\end{aligned}
$$

there exist $i_{1}, i_{2}, j_{1}$, and $j_{2}$ such that $c_{i_{1}}=M_{j_{1}}(\mathbf{c})$ and $c_{i_{2}}=M_{j_{1}}(\mathbf{c})$. Yet, either $M_{j_{1}}$ or $M_{j_{2}}$ is strict, so $c_{1}=\cdots=c_{k}$. Hence, $\{\mathbf{y}(n)\}_{n=0}^{\infty}$ converges to $\mu(x)$ e. Furthermore, $\mu(\mathbf{x})$ is squeezed between two means, so it is a mean. On the other hand, by Dini's theorem [6, page 350], $U_{n}(\mathbf{x})$ converges uniformly to $\mu(\mathbf{x})$. Since, $U_{n}$ is continuous for each $n, \mu$ is continuous. This completes the proof.

Remark 2.3. It is worth mentioning that

(i) in the literature, $\mu(\mathbf{x})$, whenever it exists, is denoted by $\otimes_{j=1}^{k} F_{j}$, particularly, when $F_{j}$ is time invariant;

(ii) also, Theorem 2.2 can be viewed as a generalization of [ 8 , Theorem 8.8$]$.

Our next result is concerned with isotone means. If $M\left(x_{1}, \ldots, x_{k}\right)$ is an isotone mean of $k$ real numbers, $L=\min \left(x_{1}, \ldots, x_{k}\right)$, and $U=\max \left(x_{1}, \ldots, x_{k}\right)$, then $M$ can be bounded by two means $\phi_{M}(L, U)$ and $\psi_{M}(L, U)$ obtained from $M$ by replacing those $x_{j}$ 's with $L<$ $x_{j}<U$ by $L$ and $U$, respectively. For example, if $k=3$ and $x_{1} \leq x_{2} \leq x_{3}$, then

$$
\phi_{A}(L, U)=\frac{2 L+U}{3} \leq A\left(x_{1}, x_{2}, x_{3}\right)=\frac{x_{1}+x_{2}+x_{3}}{3} \leq \psi_{A}(L, U)=\frac{L+2 U}{3},
$$

and so on. Now, we state and prove our second main result in this section.

Theorem 2.4. Suppose that $F_{j}$ 's are isotone means, and let $\phi_{j}(n, \cdot)$ and $\psi_{j}(n, \cdot)$ be the associated means with $F_{j}$ as defined above. If there exists a continuous strict mean $\phi$ that is dominated by $\phi_{j}(n, \cdot)$ for all $j$, then every solution $\{\mathbf{y}(n)\}_{n=0}^{\infty}$ converges to $\mu(\mathbf{x}) \mathbf{e}$, where $\mu$ is a continuous mean. A similar result holds whenever $\psi_{j}(n, \cdot)$ are dominated by a strict continuous mean $\psi$.

Proof. Let $L_{n}$ and $U_{n}$ be defined as in the proof of Theorem 2.2. Then

$$
\begin{aligned}
L_{n+1} & =\min (\mathbf{y}(n+1))=\min \left(F_{1}(n, \mathbf{y}(n)), \ldots, F_{k}(n, \mathbf{y}(n))\right) \\
& =F_{j_{n}}(n, \mathbf{y}(n)) \geq \phi_{j_{n}}\left(L_{n}, U_{n}\right) \geq \phi\left(L_{n}, U_{n}\right) \geq L_{n}, \quad \forall n .
\end{aligned}
$$


Since $L_{n} \rightarrow L, U_{n} \rightarrow U$, and $\phi$ is continuous, we have $\phi(L, U)=L$. But $\phi$ is strict, therefore $L=U$. Continuity, once again, is a consequence of Dini's theorem.

The proof in the other case is similar and will be omitted.

Our last result in this section is concerned with the case when each $F_{j}$ is periodic in the first argument, that is, there exists a positive integer $p$ such that

$$
F_{j}(n+p, \mathbf{x})=F_{j}(n, \mathbf{x}), \quad \forall j, \text { and all } \mathbf{x} .
$$

To simplify notation, we will use

$$
\begin{aligned}
\mathbf{F}_{n}(\mathbf{x}) & =\left(F_{1}(n, \mathbf{x}), \ldots, F_{k}(n, \mathbf{x})\right), \\
\mathbf{G}(\mathbf{x}) & =\mathbf{F}_{p-1} \circ \cdots \circ \mathbf{F}_{0}(\mathbf{x}) .
\end{aligned}
$$

Theorem 2.5. Suppose that there exists a positive integer $p$ such that $F_{j}(n+p, \mathbf{x})=F_{j}(n, \mathbf{x})$ for all $j$, and all $\mathbf{x}$. If the components of $\mathbf{G}$ are continuous and at least $k-1$ of them are strict, or if the components of $G$ are isotone and either $\phi$ or $\psi$ (as defined in Theorem 2.4) is continuous and strict, then every solution $\{\mathbf{y}(n)\}_{n=0}^{\infty}$ converges to $\mu(\mathbf{x}) \mathbf{e}$, where $\mu$ is a continuous mean. Furthermore, $\mu(\mathbf{x})$ is the unique mean that satisfies the functional equation

$$
\mu(\mathbf{G}(\mathbf{x}))=\mu(\mathbf{x})
$$

Proof. First, since

$$
\mathbf{y}(n p)=\mathbf{y}((n-1) p+p)=F_{p-1} \circ \cdots \circ F_{0}(\mathbf{y}((n-1) p))=G(\mathbf{y}((n-1) p)),
$$

by applying Theorems 2.2 and 2.4 to the subsequence $\{\mathbf{y}(n p)\}_{n=0}^{\infty}$, in the two situations, respectively, we obtain $U=L$, hence the convergence.

Now,

$$
\mu(\mathbf{x}) \mathbf{e}=\lim _{n \rightarrow \infty} \mathbf{y}((n+1) p)=\lim _{n \rightarrow \infty} G^{n+1}(\mathbf{x})=\lim _{n \rightarrow \infty} G^{n}(G(\mathbf{x}))=\mu(G(\mathbf{x})) \mathbf{e} .
$$

This completes the proof.

Remark 2.6. The first part of Theorem 2.5 can, also, be viewed as a generalization of [3, Theorem 8.8], and the last part is a generalization of the invariance principle in [3, page 269].

\section{Applications}

To illustrate the applicability of Theorems 2.2, 2.4, and 2.5, we present the following example.

Example 3.1. Let $\mathbf{y}(n)=\left(y_{1}(n), \ldots, y_{k}(n)\right)$ such that $\mathbf{y}(0)=\left(x_{1}, \ldots, x_{k}\right) \in \mathbb{R}_{+}^{k}$, and $F_{j}(n$, $\mathbf{y}(n))=H_{p_{j}}(n, O(\mathbf{y}(n)))$, where $O$ is the sorting operator that arranges $\mathbf{y}(n)$ in ascending order. Then, we have the iterative process

$$
y_{j}(n+1)=H_{p_{j}}(n, O(\mathbf{y}(n))), \quad j=1, \ldots, k .
$$


Now, one may think of many possible scenarios where Theorems 2.2, 2.4, and 2.5 can be applied. In particular, if $H_{p_{j}}(n, \mathbf{x})=\left(\sum_{i=1}^{k} w_{i j}(n) x_{i}^{p_{j}}\right)^{1 / p_{j}}$, and $c=\inf _{n} \min _{i, j} w_{i j}(n)>0$, then with

$$
\begin{gathered}
Z=\{1, \ldots, k\}, \\
L_{n}=\min _{i \in Z} y_{i}(n)=y_{k_{n}}(n) \quad \text { for some } k_{n} \in Z, \\
U_{n}=\max _{i \in Z} y_{i}(n)=y_{\ell_{n}}(n) \quad \text { for some } \ell_{n} \in Z, \\
I_{n}=\left\{i \in Z: y_{i}(n)=L_{n}\right\}, \\
J_{n}=\left\{i \in Z: y_{i}(n)=U_{n}\right\}, \\
K_{n}=Z \backslash\left(I_{n} \cup J_{n}\right),
\end{gathered}
$$

we have

$$
\begin{aligned}
L_{n+1}^{p_{n+1}}= & {\left[y_{k_{n+1}}(n+1)\right]^{p_{k_{n+1}}}=\sum_{j=1}^{k} w_{j, k_{n+1}}(n)\left[y_{j}(n)\right]^{p_{k_{n+1}}} } \\
= & \left(\sum_{j \in I_{n}} w_{j, k_{n+1}}(n)\right) L_{n}^{p_{k_{n+1}}}+\left(\sum_{j \in J_{n}} w_{j, k_{n+1}}(n)\right) U_{n}^{p_{k_{n+1}}} \\
& +\left(\sum_{j \in K_{n}} w_{j, k_{n+1}}(n)\left[y_{j}(n)\right]^{p_{k_{n+1}}}\right) \\
\geq & \left(\sum_{j \in I_{n} \cup K_{n}} w_{j, k_{n+1}}(n)\right) L_{n}^{p_{k_{n+1}}}+\left(\sum_{j \in J_{n}} w_{j, k_{n+1}}(n)\right) U_{n}^{p_{k_{n+1}}} \\
= & L_{n}^{p_{k_{n+1}}}+\left(\sum_{j \in J_{n}} w_{j, k_{n+1}}(n)\right)\left(U_{n}^{p_{k_{n+1}}}-L_{n}^{p_{k_{n+1}}}\right) \geq L_{n}^{p_{k_{n+1}}}+c\left(U_{n}^{p_{k_{n+1}}}-L_{n}^{p_{k_{n+1}}}\right) \\
= & (1-c) L_{n}^{p_{k_{n+1}}}+c U_{n}^{p_{k_{n+1}}},
\end{aligned}
$$

and so

$$
L_{n+1} \geq\left[(1-c) L_{n}^{p_{k_{n+1}}}+c U_{n}^{p_{k_{n+1}}}\right]^{1 / p_{k_{n+1}}}
$$

Thus, if $p_{j}=p$ for all $j$, then

$$
L_{n+1} \geq\left[(1-c) L_{n}^{p}+c U_{n}^{p}\right]^{1 / p}
$$

and so Theorem 2.4 is applicable. Furthermore, if $w_{i j}$ 's are independent of $n, W=\left[w_{i j}\right] \in$ $\mathbb{R}_{+}^{k \times k}, \mathbf{x}^{p}=\left(x_{1}^{p}, \ldots, x_{k}^{p}\right)$, and $\mathbf{z}(n)=\mathbf{y}^{p}(n)$, then

$$
\mathbf{z}(n+1)=W \mathbf{z}(n),
$$


and so

$$
\tilde{\mu}(W \mathbf{z}(0))=\tilde{\mu}(\mathbf{z}(0)), \quad \tilde{\mu}=\mu^{p} .
$$

Substituting

$$
\tilde{\mu}(\mathbf{t})=\sum_{i=1}^{k} \lambda_{i} t_{i}, \quad \lambda_{i} \geq 0, \sum_{i=1}^{k} \lambda_{i}=1,
$$

we reach the conclusion that $\lambda=\left(\lambda_{1}, \ldots, \lambda_{k}\right)^{T}$ satisfies

$$
W^{T} \boldsymbol{\lambda}=\boldsymbol{\lambda}
$$

that is, $\lambda$ is a normalized nonnegative eigenvector associated with 1 , if there is any. But the existence of such an eigenvector is assured by the celebrated Perron's theorem [7, page 503]. Hence,

$$
\mu(\mathbf{x})=\left[\sum_{i=1}^{k} \lambda_{i} \tilde{x}_{i}^{p}\right]^{1 / p}, \quad \tilde{\mathbf{x}}=O(\mathbf{x})
$$

Remark 3.2. It is worth mentioning that it is possible to formulate a weaker condition on the weights $w_{i j}$ 's that ensures convergence. Also, by taking $p=1$ and $0+$, we obtain results about compounding arithmetic means and geometric means, respectively. Indeed, in the later case, by L'Hôpital's rule,

$$
\begin{aligned}
\mu(\mathbf{x}) & =\lim _{p \rightarrow 0+} \exp \left(\frac{\ln \left(\sum_{i=1}^{k} \lambda_{i} \tilde{x}_{i}^{p}\right)}{p}\right)=\lim _{p \rightarrow 0+} \exp \left(\frac{\sum_{i=1}^{k} \lambda_{i} \tilde{x}_{i}^{p} \ln \left(\tilde{x}_{i}\right)}{\sum_{i=1}^{k} \lambda_{i} \tilde{x}_{i}^{p}}\right) \\
& =\exp \left(\sum_{i=1}^{k} \lambda_{i} \ln \left(\tilde{x}_{i}\right)\right)=\prod_{i=1}^{k} \tilde{x}_{i}^{\lambda_{i}} .
\end{aligned}
$$

\section{Conclusion}

In this research, we investigated the convergence of solutions of nonautonomous dynamical systems driven by means. In particular, when all the components converge to a common limit. In light of Lemma 2.1 and the results established in [1], we formulate the following conjecture.

Conjecture 4.1. If $\{\mathbf{y}(n)\}_{n=0}^{\infty}$ with $\mathbf{y}(0)=\mathbf{x}$ a solution of $(1.1)$, then the limit set $w(\mathbf{x})=$ $\left\{\mathbf{z} \mid \lim _{i \rightarrow \infty} \mathbf{y}\left(n_{i}\right)=\mathbf{z}\right\}$ is finite.

\section{References}

[1] R. M. Abu-Saris, Asymptotic behavior of discrete processes driven by means, Nonlinear Analysis 59 (2004), no. 1-2, 245-251.

[2] R. M. Abu-Saris and M. Hajja, Quadratic means, Journal of Mathematical Analysis and Applications 288 (2003), no. 1, 299-313. 
[3] J. M. Borwein and P. B. Borwein, Pi and the AGM, Canadian Mathematical Society Series of Monographs and Advanced Texts, vol. 4, John Wiley \& Sons, New York, 1998.

[4] P. S. Bullen, Handbook of Means and Their Inequalities, Mathematics and Its Applications, vol. 560, Kluwer Academic, Dordrecht, 2003.

[5] W. Cheney, Analysis for Applied Mathematics, Graduate Texts in Mathematics, vol. 208, Springer, New York, 2001.

[6] S. N. Elaydi, Nonautonomous difference equations: open problems and conjectures, Differences and Differential Equations, Fields Inst. Commun., vol. 42, American Mathematical Society, Rhode Island, 2004, pp. 423-428.

[7] R. A. Horn and C. R. Johnson, Matrix Analysis, Cambridge University Press, Cambridge, 1985.

[8] R. Kempf, On $\Omega$-limit sets of discrete-time dynamical systems, Journal of Difference Equations and Applications 8 (2002), no. 12, 1121-1131.

[9] J. Matkowski, Iterations of mean-type mappings and invariant means, Annales Mathematicae Silesianae (1999), no. 13, 211-226.

Raghib M. Abu-Saris: Department of Basic Sciences, University of Sharjah, P.O. Box 27272,

Sharjah, United Arab Emirates

E-mail address: rabusaris@sharjah.ac.ae 